\title{
Médiévales
}

Langues, Textes, Histoire

57 | automne 2009

Langages politiques, $\mathrm{XII}{ }^{\mathrm{e}}-\mathrm{XV}^{\mathrm{e}}$ siècle

\section{Principat, communauté et individu au bas Moyen Âge. Cultures politiques dans l'État de Milan}

Princedom, Community and the Individual in the Late Middle Ages. Political

Cultures in the State of Milan

\section{Massimo Della Misericordia}

Traducteur : Laurence Moulinier-Brogi

\section{OpenEdition}

\section{Journals}

Édition électronique

URL : https://journals.openedition.org/medievales/5807

DOI : $10.4000 /$ medievales. 5807

ISSN : 1777-5892

Éditeur

Presses universitaires de Vincennes

\section{Édition imprimée}

Date de publication : 20 décembre 2009

Pagination : 93-111

ISBN : 978-2-84292-241-2

ISSN : 0751-2708

Référence électronique

Massimo Della Misericordia, «Principat, communauté et individu au bas Moyen Âge. Cultures politiques dans l'État de Milan », Médiévales [En ligne], 57 | automne 2009, mis en ligne le 18 janvier 2012, consulté le 22 avril 2022. URL : http://journals.openedition.org/medievales/5807 ; DOI : https:// doi.org/10.4000/medievales.5807 
Médiévales 57, automne 2009, p. 93-112

Massimo DELLA MISERICORDIA

\section{PRINCIPAT, COMMUNAUTÉ ET INDIVIDU AU BAS MOYEN ÂGE. CULTURES POLITIQUES DANS L'ÉTAT DE MILAN}

Le présent article est la partie conclusive d'une recherche sur le débat politique qui s'est développé dans la Lombardie du $\mathrm{XV}^{\mathrm{e}}$ siècle autour de la possibilité, pour la communauté locale considérée dans son unité, de s'exprimer, de décider et d'agir, de s'attribuer elle-même des actions déterminées (qu'elle ne peut à l'évidence pas accomplir en tant que telle, comme le fait de parler), au moment où elle en confère la responsabilité à des représentants. Dans des travaux précédents, j'ai examiné le langage des sources avant tout dans le but de discuter la question de la vitalité des idéaux républicains à l'intérieur d'une principauté italienne de la Renaissance, et celle de la constitution, encouragée par les pouvoirs centraux, d'élites locales plus sélectives dans les régimes politiques du Moyen Âge tardif ${ }^{1}$. Ce qui m'intéresse principalement ici, c'est de revisiter une thèse classique, à savoir d'examiner si les pouvoirs plus centralisés qui se constituent à l'époque de la Renaissance italienne ont légitimé, via une polémique culturelle contre les communautés et la promotion des valeurs de la distinction sociale en leur sein, l'affirmation de l'individu comme protagoniste politique.

Selon moi, lors de la domination des Visconti et des Sforza, le principat, appliqué à forger des sentiments nouveaux de loyauté et des nouveaux critères de légitimation, se posa en polémique ouverte face aux idéaux élabo-

1. «Decidere e agire in comunità nel XV secolo (un aspetto del dibattito politico nel dominio sforzesco) », dans A. GamberinI, G. Petralia dir., I linguaggi politici nell'Italia del Rinascimento (s. XIV-XV), Rome, 2007, p. 293-380 ; «Como se tuta questa universitade parlasse. Rappresentare la comunità di fronte al principe nello stato di Milano (s. XV) », dans F. ForondA dir., Avant le contrat social... Le contrat politique dans l'Occident médiéval (XIII ${ }^{e}-X V^{e}{ }^{e}$.), Paris (en préparation). Une discussion historiographique plus ample sera développée ailleurs. Pour l'heure, je renvoie en particulier aux références bibliographiques des deux textes, d'où sont tirées aussi les citations des sources desquelles n'est pas précisée la cote dans les archives. 
rés en particulier au cours du XIII ${ }^{\mathrm{e}}$ siècle, qui avaient placé la commune au centre des relations politiques. Dans la documentation gouvernementale dont cet article se propose d'étudier le langage (à l'exclusion des sources de nature littéraire ou de la littérature de traités), la chancellerie des ducs et leur entourage offrent un riche éventail de thèmes. Partant du présupposé de la «qualité » irréductiblement diverse des membres de la communauté (gentilshommes ou vilains, principali ou artisans «minimes »), ils frappèrent la communauté même au moins de deux points de vue : les formes de la participation politique au niveau local et la possibilité d'accéder au prince en tant que collectivité. Bien que la politique des seigneurs de Milan ait investi tant les communautés urbaines que rurales, ce sont surtout ces dernières, plus étrangères aux normes de comportement social et politique prescrites par les autorités étatiques, qui furent exposées au risque de délégitimation politique. L'issue fut en effet la promotion du rôle politique de l'individu, connoté par son status et sa réputation personnelle. Les corps territoriaux, toutefois, en particulier les ruraux, avec le concours de leurs élites, ne subirent pas passivement une remise en cause aussi agressive de leur subjectivité politique, et restèrent fidèles aux pratiques et aux rhétoriques de la cohésion communautaire et de l'intégration des individus dans l'unité définie de manière institutionnelle.

\section{Contre la forme. L'autorité du prince, les institutions communautaires, la qualité individuelle}

Dans l'Italie septentrionale, entre le $\mathrm{XI}^{\mathrm{e}}$ et le $\mathrm{XIII}{ }^{\mathrm{e}}$ siècle, on assista à une floraison d'expériences d'autogouvernement urbain et rural, auxquelles se superposèrent, à partir de la fin du XIII ${ }^{\mathrm{e}}$, des pouvoirs seigneuriaux exercés sur des ensembles plus étendus. L'incorporation dans des états dits régionaux ou territoriaux des formations politiques, avec les valeurs et les pratiques connexes qui s'étaient développées aux siècles précédents - les seigneuries locales, les factions, les communes urbaines et rurales -, ne signifia pas la substitution pure et simple d'une forme institutionnelle à une autre, mais détermina une rencontre singulière entre des cultures différentes. Avec ces sujets enracinés dans le territoire et légitimés par la coutume et par le droit, le pouvoir princier dut de fait ouvrir une négociation complexe, qui comporta tantôt légitimation réciproque, tantôt incompréhension, tantôt encore conflit. En particulier, le régime communal ne s'éteignit pas, mais continua à être la forme de gouvernement de ces sujets qui, ayant perdu la libertas, étaient toutefois reconnus par le pouvoir central comme des corps territoriaux au sein de leur domination, liés au prince ou, dans les républiques, aux villes dominantes, par un pacte qui les engageait à l'obéissance, mais qui sauvegardait aussi des marges d'autonomie ${ }^{2}$.

2. A. De Benedictis, Repubblica per contratto. Bologna: una città europea nello Stato della Chiesa, Bologne, 1995. 
Parmi les différentes interprétations de l'État italien à la fin du Moyen Âge, on peut proposer par conséquent d'y voir une stratigraphie de cultures politiques, qui reconnaisse la coexistence et la concurrence entre des pratiques et des langages anciens et récents ${ }^{3}$. Un des motifs de plus âpre friction entre ces différentes strates culturelles et les acteurs qui s'en réclamaient concerna précisément le rôle de la communauté. Au XIII ${ }^{\mathrm{e}}$ siècle, en effet, on en était arrivé à préciser sous le profil idéologique le sujet communal, une institution gouvernée par des normes écrites et des organismes formalisés, ayant pour fin la poursuite du «bonum commune». S'étaient alors précisés les mécanismes électoraux, les règles du débat et de la décision en conseil qui permettaient une participation politique large à la population en tant que collectivité organisée ${ }^{4}$. Le même gouvernement du territoire vit la responsabilisation, juridiquement définie, de la commune rurale face à la commune urbaine, pour autant que cela concernait le maintien de la paix territoriale, l'entretien des infrastructures routières, le paiement des charges fiscales, et ainsi de suite.

Ce modèle fut attaqué de plusieurs côtés par l'affirmation des états régionaux, avec le succès des nouvelles valeurs sociales d'inspiration aristocratique et de différents programmes politiques, caractérisés par l'autoritarisme, ainsi que, en lien étroit avec ce dernier, par la préférence pour des dispositifs informels de gouvernement plutôt que pour les procédures à haut degré de définition juridique. Par rapport au degré élevé de formalisation de la politique communale et au solide encadrement juridique du pouvoir attesté notamment au XIII ${ }^{\mathrm{e}}$ siècle, les républiques oligarchiques et encore plus les gouvernements princiers de la fin du Moyen Âge se servirent largement de moyens « extraordinaires » et extra-légaux. En ce qui concerne les rapports entre États, la diplomatie officielle s'accompagna de canaux réservés et confidentiels. Dans le domaine du droit, les autorités gouvernantes se réservèrent un pouvoir normatif qui pouvait innover par rapport à la coutume. En outre, la norme elle-même pouvait être l'objet d'exceptions selon la volonté du prince. Aux modèles de procès mis au point au XIII ${ }^{\mathrm{e}}$ siècle, hautement définis sous le profil procédural même au moment où il s'agissait de poursuivre les ennemis politiques du régime, s'ajoutèrent des interventions sommaires, dans lesquelles l'arbritrium du seigneur et du magistrat qui le représentait avait largement cours. Pour diriger les institutions locales, plutôt que des podestats tenus au respect des statuts locaux, furent envoyés des commissaires dotés d'une autorité aux marges moins précises, et dont les actes de gouvernance étaient moins tenus par le respect du droit ${ }^{5}$.

3. M. Folin, Rinascimento estense. Politica, cultura, istituzioni di un antico stato italiano, Rome-Bari, 2001.

4. M. Aschieri, E. Artifoni, G. Milani, «Il governo delle città nell'Italia comunale. Una prima forma di democrazia ?», Bollettino roncioniano, VI, 2006, p. 5-49. Cf. M. VALLERANI, La giustizia pubblica medievale, Bologne, 2005.

5. A. ZoRZI, L'amministrazione della giustizia penale nella Repubblica fiorentina. Aspetti e problemi, Florence, 1988 ; R. FubINI, Italia quattrocentesca. Politica e diplomazia nell'età di Lorenzo il Magnifico, Milan, 1994. Voir récemment A. GAMBERINI, La città assediata. Poteri e 
L'instauration de ces pratiques de gouvernement accompagna des transformations dans les classifications sociales et en général dans les rapports de pouvoir, elles aussi dans le sens d'une plus grande informalité. La taxonomie des status abandonna les cadres de classes, plus rigides, accrédités par le passé par les sources normatives et notariales (qui identifiaient voisins, citoyens et nobles, ou, au $\mathrm{XV}^{\mathrm{e}}$, «gentilshommes »); en revanche, la notion fluide de qualitas individuelle se mit à prendre de l'importance, en d'autres termes, la position qu'occupait une personne par sa naissance, sa richesse ou son prestige ${ }^{6}$. « Meliores » et surtout « principali » deviendront les mots-clés de la lecture plus ductile de la société par les nouveaux gouvernants; leur but était de délimiter des profils plus personnalisés, d'identifier combien de personnes, indépendamment de l'inscription dans les ordres définis sur une base juridique et coutumière, exerçaient une influence locale décisive et pouvaient constituer des interlocuteurs directs pour le gouvernement du pays, éventuellement au-delà des relations institutionnelles entretenues entre le prince et les corps territoriaux.

Dans ce contexte, de nombreuses formations de type corporatif (des communitates de classe aux associations de métiers, des contrade urbaines aux universitates de faction) durent affronter une grave crise de légitimité. Certaines d'entre elles furent reconnues par les nouvelles autorités centrales, mais soumises à contrôle, d'autres furent marginalisées, d'autres encore furent combattues. En particulier, le rapport entre le gouvernement princier et les communes rurales et urbaines fut ambivalent. L'habitude qu'ont les officiers des Sforza et la chancellerie de l'État d'éviter, bien que sans censure systématique, le terme "commune », un mot qui à d'autres époques aussi et au sein dautres régimes avait suscité l'inquiétude ${ }^{7}$, est emblématique de ce phénomène : c'est l'indice de la volonté ducale de légitimer de tels sujets à condition qu'ils acceptent un profil politique bas. En effet les communes eurent un rôle reconnu dans la constitution de l'État territorial, mais leur orgueil autonomiste et le caractère peu contrôlable de leur débat donnaient du souci à qui était chargé d'obtenir l'exécution des ordres des Sforza ; leurs procédures étaient un obstacle à la pratique du pouvoir extra-légal que les

identità politiche a Reggio in età viscontea, Rome, 2003 ; L. TANZINI, Il governo delle leggi. Norme e pratiche delle istituzioni a Firenze dalla fine del Duecento all'inizio del Quattrocento, Florence, 2007 ; N. CovinI, «La balanza drita ». Pratiche di governo, leggi e ordinamenti nel ducato sforzesco, Milan, 2007.

6. M. Della Misericordia, Divenire comunità. Comuni rurali, poteri locali, identità sociali e territoriali in Valtellina e nella montagna lombarda nel tardo medioevo, Milan, 2006, partie II.

7. O. G. OEXLE, «Gilde und Kommune. Über die Entstehung von "Einung" und "Gemeinde" als Grundformen des Zusammenlebens in Europa », dans P. BLICKLE dir., Theorien kommunaler Ordnung in Europa, Munich, 1996, p. 75-97 ; O. BANTI, "Civitas" e "Commune" nelle fonti italiane dei secoli XI e XII », dans G. RosSETTI dir., Forme di potere e struttura sociale in Italia nel medioevo, Bologne, 1977, p. 217-232 ; G. Vitolo, «In palatio Communis. Nuovi e vecchi temi della storiografia sulle città del Mezzogiorno medievale», dans G. ChitTolini, G. PetTI Balbi, G. Vitolo dir., Città e territori nell'Italia del Medioevo. Studi in onore di Gabriella Rossetti, Naples, 2007, p. 243-294. 
ducs tendaient à affirmer. Les communautés, en protestant contre les facultés de gouvernement arbitraire que s'étaient réservées les seigneurs de Milan, dénonçaient toute prétention du prince qui se situait « contra la forma de loro capituli et privilegii », ou contre les statuts ${ }^{8}$. Les princes, dans ces occasions, cherchaient tout de même le dialogue avec le pays, mais souvent par l'entremise des éléments les plus fiables parmi les personnes de plus haute distinction sociale, qui œuvraient au sein, aux marges, et parfois à la place des magistratures communautaires locales, que le nouveau vocabulaire de l'excellence dont on a parlé plus haut permettait de reconnaître.

\section{Les intermédiaires du langage : les lettres}

Il faut observer que la nature même des sources conservées pour le $\mathrm{XIII}^{\mathrm{e}}$ siècle ne permet pas, en général, d'entrer dans le vif des phénomènes de la médiation clientélaire et de la relation entre gouvernants et interlocuteurs locaux puissants mais non institutionnels, qui nous sont connus grâce aux riches correspondances $d u \mathrm{XV}^{\mathrm{e}}$ siècle. Je ne pense pas, toutefois, que ce silence invalide la possibilité d'une périodisation de longue durée des cultures politiques, puisque la typologie de la documentation transmise n'est pas indifférente aux valeurs et aux pratiques du gouvernement. De fait, la rupture des carcans formulaires et typologiques de la documentation communale opérée par la lettre viscontéo-sforzesque (comme celle d'autres dominations de la Péninsule), avec la grande ouverture de sa teneur dispositive et narrative par rapport à l'épistolographie des régimes précédents, a pour correspondant, sur le plan de la communication politique, le passage des relations dûment institutionnalisées que le gouvernement communal établissait avec le territoire, à la gamme de voies formelles et informelles avec lesquelles l'État régional atteignait les périphéries.

La commune urbaine codifia ses propres rapports vis-à-vis des centres qui lui étaient soumis avec les termes normatifs des statuts. L'interaction quotidienne, en outre, se déroulait principalement grâce aux instruments notariaux ou de secs mandats judiciaires. Dans le panorama de l'épistolographie des communes lombardes, un corpus édité récemment se signale : il s'agit des lettres émanant entre 1263 et 1299 des juges et assesseurs du podestat communal ou des vicaires du seigneur de Brescia. Elles ordonnaient («precipiendo mandamus », « iungimus ») aux communes, aux hommes et à leurs officiers de différentes terres du contado la description des propriétés et des loyers contestés, des mises en possession, des saisies et séquestres des récoltes, etc.

8. Archivio di Stato di Milano [ASMi], Comuni, 60, Morbegno, s.d. Cf. M. Della MisericoRdiA, «Per non privarci de nostre raxone, li siamo stati desobidienti. Patto, giustizia e resistenza nella cultura politica delle comunità alpine nello stato di Milano (s. XV) », dans C. NubOLA, A. WÜRGLER dir., Forme della comunicazione politica in Europa nei secoli XV-XVIII. Suppliche, gravamina, lettere, Bologne, 2004, p. 147-215. 
Ce sont des écrits qui se présentent, de manière immédiate, comme dénués de la variété des missives destinées par les Sforza aux sujets politiques locaux, et davantage assimilables, par le contenu et les objectifs, au précepte judiciaire ${ }^{9}$.

Les Visconti et les Sforza, de leur côté, ne définirent jamais avec l'organicité d'un texte statutaire les responsabilités ou les prérogatives des communes rurales et urbaines face à l'État. Cette matière était régulée par des statuts locaux dont la validité fut confirmée, par une myriade de privilèges particuliers et par la négociation entre gouvernants et gouvernés engendrée par chaque imposition fiscale ou militaire. Un document en particulier se fit le véhicule de telles pratiques négociées, la «littera clausa », dont l'usage devint massif avec le passage du régime communal au régime seigneurial. Bien que la composition de tels textes épistolaires fût guidée par une tradition rhétorique et documentaire, ils se signalent par une variété du vocabulaire et une élasticité de leur structure plus grandes que celles des documents notariés et judiciaires. C'est à eux qu'étaient confiés les instructions et les ordres envoyés par le prince vers la périphérie, les rapports de ses officiers, les réponses des communautés, les demandes des principali et ainsi de suite. La souplesse de la structure formelle, et la possibilité qui s'ensuivait d'articuler de manière indéfinie les contenus de l'écrit, furent donc le medium des nouveaux mécanismes de gouvernement, plus flexibles ${ }^{10}$. Ils permirent donc au duc, à la chancellerie et aux officiers périphériques d'échanger des suggestions dans la recherche d'un rapport avec les «meilleurs » en marge des canaux institutionnels, des observations confidentielles sur les principali, des instructions qu'il aurait été matériellement impossible de donner au moyen de la documentation, si rigide dans sa composition, du XIII ${ }^{\mathrm{e}}$ siècle, et qui en vérité, au XIV ${ }^{\mathrm{e}}$ siècle encore, n'apparaissent pas si subtilement articulées qu'au siècle suivant ${ }^{11}$.

Les mêmes communautés locales valorisèrent la nature d'écriture politique, plutôt que probatoire, de la lettre, tandis que sa composition était confiée par exemple au maître d'école, expert en rhétorique, et au dictamen des officiers en exercice, plutôt qu'à un simple notaire qui dominait surtout le formulaire juridique ${ }^{12}$.

9. P. MERATI, « Comunicare con il contado. Le lettere dei rettori di Brescia ai comuni rurali (XIII s.) », dans L. ChIAPPA MAURI dir., Contado e città in dialogo. Comuni urbani e comunità rurali nella Lombardia medievale, Milan, 2003, p. 141-170. Cf. M. Della MisericordiA, « Mappe di carte. Le scritture e gli archivi delle comunità rurali della montagna lombarda nel basso medioevo », dans A. Bartoli Langeli, A. Giorgi, S. Moscadelli dir., Archivi e comunità tra Medioevo ed Età Moderna, Rome-Trente, 2009, p. 212-215.

10. A. Bartoli LANGeli, «La documentazione degli Stati italiani nei secoli XIII-XV : forme, organizzazione, personale », dans Culture et idéologie dans la genèse de l'État moderne, Rome, 1985, p. 35-55 ; I. LAZZARINI, " Materiali per una didattica delle scritture pubbliche di cancelleria nell'Italia del Quattrocento », Scrineum-Rivista, 2 (2004), p. 15 sq. ; A. GambERINI, Lo stato visconteo. Linguaggi politici e dinamiche costituzionali, Milan, 2005, p. 40-52.

11. I « registri litterarum » di Bergamo (1363-1410). Il carteggio dei signori di Bergamo, P. Mainoni, A. SALA éd., Milan, 2003.

12. Archivio storico del comune di Bormio, Quaterni consiliorum, 4, 1504.04.12. 


\section{Communauté, gentilshommes, vilains}

Les lettres émanant des pouvoirs centraux et des magistratures périphériques de l'État furent non seulement des instruments cruciaux de gouvernement, mais aussi les véhicules d'une polémique idéologique vis-à-vis de la tradition communale.

Une première forme de délégitimation culturelle et politique des pratiques locales de participation et d'assemblées recourut aux valeurs aristocratiques, alimentées par une sensibilité éclectique (chevaleresque, urbaine et humaniste). L'histoire politique, en effet, ne peut négliger le fait que les rapports institutionnels, juridictionnels, économiques, entre la ville et le contado, entre le prince et la communauté, s'inséraient, à l'époque considérée ici, dans un contexte largement dominé par la dépréciation, à la ville et à la cour, du paysan, des ville (villages) dans lesquelles il habitait, et des codes de conduite qui y avaient cours. La littérature de traités, les nouvelles et l'historiographie caractérisaient leurs protagonistes de manière appuyée, en offrant une taxonomie sociale précise qui distinguait le citadin, le gentilhomme, le vilain, souvent obtus ou rusé, ingrat, orgueilleux, et bagarreur ${ }^{13}$. La dégradation sociale servit à marginaliser plus d'une expérience politicoinstitutionnelle : les corporations d'artisans, par exemple, furent mises à l'écart par les gouvernements urbains à cause de la «vileté »- aux yeux des élites citadines de gouvernement - des activités des catégories qu'elles représentaient. Les communautés rurales, en particulier, durent traiter avec les officiers centraux et, souvent, périphériques, qui s'étaient formés au milieu de ces préjugés aristocratiques et urbains. Et bien qu'on manque encore d'une étude systématique sur la culture de la bureaucratie ducale, les échos de la satire du vilain apparaissent évidents dans la dénonciation, de la part des officiers, de la conduite «téméraire » et «présomptueuse » des popolari, dans leur manière de réduire la vie publique des communautés rurales au «tumulte » et au « désordre », dans leur mépris pour le «popullo vulgare et grosso » qui «n'entend pas ».

De cette manière, les autorités étatiques posèrent les conditions, non seulement d'une dégradation sociale des sphères d'organisation politiques peu contrôlables, mais aussi d'une méconnaissance plus ambitieuse du profil unitaire des corps locaux, de leur consistance institutionnelle et de leur subjectivité politique. Les deux moments fondamentaux de l'expression des communautés - la réunion des chefs de famille ou des conseillers et l'institution de syndics destinés à agir et parler au nom de leurs voisins - en furent investis. Les agents du principat distinguèrent de manière emphatique les qualités de leurs membres : les principali, les «gentilshommes », les «boni cives », et enfin les personnes d'humble extraction ou les «vilains». Ces derniers étaient considérés comme incapables d'autonomie de jugement et 
d'initiative, de même que privés de la pleine dignité politique. J'examinerai dans les pages qui suivent les implications de ces préventions sociales sur le remodelage des conditions de la représentation. Pour le moment je relève que, étant donné ces prémisses, le prince et les officiers d'état étaient autorisés à ne plus considérer un conseil en tant que tel, mais à le soumettre à un tri susceptible de distinguer gentilshommes et vilains, ou encore à décomposer la communauté en principali collaborateurs, criminels à punir pour leur désobéissance, et popolari à l'esprit grégaire.

De manière très éloquente, même l'instruction produite à la cour et consacrée à la formation du jeune Galeazzo Maria Sforza, qui éduquait le futur prince à établir des rapports avec les villes et les terres de la domination ancestrale qu'il allait visiter, l'invitait à une connaissance par le menu des sites, des fortifications et des «homini [...] de reputatione », sans prévoir aucun contact avec les organismes locaux qui le gouvernaient ${ }^{14}$. Il devenait alors conséquent pour les gouvernants de tenir peu de compte du débat en conseil, du vote, de l'unanimité et des autres valeurs de la tradition communale, pour préférer les tractations avec des groupes assez restreints d'hommes, quelques notables de prestige avec lesquels le dialogue serait plus aisé, grâce aussi à une syntonie culturelle plus grande, et en éludant les procédures formalisées de la vie politique locale.

\section{Péchés et passions}

Une autre ressource, dans la bataille engagée par les autorités milanaises, fut le langage moral et religieux, qui identifiait les péchés de la vie publique locale. Encore une fois, il s'agit de moyens culturels expérimentés aussi par le principat dans la compétition avec les autres sujets politiques qui l'avaient précédé : les factions en particulier, au cours du $\mathrm{XV}^{\mathrm{e}}$ siècle, furent discréditées au moyen d'un vocabulaire qui assimilait l'adhésion à un parti à un péché et à la manifestation de passions négatives ${ }^{15}$.

Dans la typologie des sept péchés capitaux qui eut une grande fortune au Moyen Âge, trois en particulier se prêtaient à la caractérisation des défauts de la coexistence organisée : l'envie, la colère et l'orgueil ${ }^{16}$. Bien représentés dans la littérature de traités et dans le discours politique produit par ces mêmes communautés, qui en percevaient le danger, ils furent utilisés par les

14. M. FeRRARI, "Per non manchare in tuto del debito moi ». L'educazione dei bambini Sforza nel Quattrocento, Milan, 2000, p. 78.

15. M. Gentile, «Discorsi sulle fazioni, discorsi delle fazioni. "Parole e demonstratione partiale" nella Lombardia del secondo Quattrocento », dans GAMBERINI, PETRALIA dir., I linguaggi politici, p. 381-408; S. FERENTE, Gli ultimi guelfi. Passioni e identità politiche nell'Italia del secondo Quattrocento, tesi di dottorato di ricerca, Istituto universitario europeo, Florence, 2007.

16. C. Casagrande, S. Vecchio, I sette vizi capitali. Storia dei peccati nel Medioevo, Turin, 2000 (trad. fr., Paris, 2002). 
officiers des Sforza pour une description de la politique locale qui en impliquait la condamnation.

L'envie était mère de discordes, dans les questions de nature privée (pour des raisons d'héritage et de patrimoine), mais aussi dans le débat public. Quand les hommes étaient divisés par «invidie e guerre asay », la réunion annuelle des chefs de famille devenait un moment intrinsèquement périlleux selon le podestat, qui s'attendait à ce qu'elle dégénère en violence ${ }^{17}$.

La colère était, comme l'envie, un péché engendré dans les rapports que les sujets entretenaient entre eux sur un plan horizontal. Elle opposait une communauté à une autre dans les litiges territoriaux ou un individu à son voisin, polluant ainsi la vie publique. Mais elle pouvait intéresser aussi la relation politique verticale, entre gouvernants et gouvernés ; " grande ira » opposait un commissaire et une famille de notables ; les sujets étaient « in iram ellevati » contre un feudataire et le duc lui-même ${ }^{18}$.

L'orgueil est le péché qui menaçait plus directement ce que le duc et ses hommes considéraient comme la normalité des rapports entre supérieur et inférieur, suscitant des formes l'insubordination individuelle ou collective. «Superbi et inhobedienti », tels pouvaient être tant des nobles que des vilains ou toute une population (la « superbia del payxe »), et d'une manière générale la personne ou la collectivité qui refusait sa propre position de gouverné («questi homeni montarano in tanta superbia che non ce porà stare officiale alcuno, né se porano gubernare ${ }^{19}$ ). Insolents et téméraires, pour finir, étaient les membres de l'assemblée et le conseil tout entier qui prétendait débattre les questions d'actualité sans la tutelle du représentant des Sforza et péchait ainsi par «presontione».

La réprobation de l'affectivité déréglée était liée à cette tradition. Certains sentiments étaient tenus pour positifs, en premier lieu «l'amore» et «l'affectione» des sujets pour le prince; à l'opposé, et toujours négatif, on trouvait au contraire «l'appetito disordinato ${ }^{20}$. Les autorités étatiques, encore une fois, dans la polémique qui les opposait aux factions, s'approprièrent la réflexion scolastique, qui permettait d'assimiler amor inordinatus et péché ${ }^{21}$, et pouvait tout à fait s'accorder avec les préceptes humanistes, marquée de l'empreinte des classiques, dans la prescription d'une éthique du sujet qui idéalisait la manifestation retenue des passions.

Or l'assemblée des chefs de famille et les conseils communautaires étaient des moments problématiques. Ils représentaient une intensification notable de l'expérience relationnelle, communicative (on s'y exprimait non

17. ASMi, Carteggio Sforzesco [CS], 720, 1463.01.12.

18. ASMi, CS, 781, 1467.02.14; 1157, 1497.09.03.

19. ASMi, CS, 718, 1454.07.08; 720, 1462.01.22; 1157, 1497.05.05.

20. $C f$. B. H. Rosenwein, «Émotions en politique. Perspectives de médiéviste », dans Hypothèses 2001, Paris, 2002, p. 315-324.

21. Gentile, Discorsi sulle fazioni, p. 406. 
seulement avec les mots mais avec les corps, en manifestant par exemple opposition ou contrariété face à une proposition en se levant ou en s'asseyant ${ }^{22}$ ), et sensorielle (le son de la cloche qui convoquait les participants, l'écoute des discours des autres). Une telle expérience connaissait parfois une véritable exaspération émotionnelle, avec les «parole matte » que l'on prononçait et qui pouvaient devenir un «strepito di parole », les injures échangées par les participants, les discours exposés «con le lacrime a li ochii », les armes que l'on montrait ${ }^{23}$.

Les gardiens du «bon ordre » pour le compte du prince purent donc faire des conseils, à plus forte raison ceux qui étaient les plus fréquentés et les moins sélectifs, le catalyseur des mouvements de l'âme potentiellement incontrôlables et obscurs. L' «affectione che portano [...] in diversitate » conduisait les hommes à d'interminables affrontements internes. Le podestat de Bormio, Gottardo Togio, reconnaissait les décisions prises dans le conseil du bourg «cum rasone » et «senza alcuni rangori », idéalisant ainsi un modèle qui contrastait avec une pratique du conseil orientée par les «apetiti » ${ }^{24}$. Lui le premier, pour avancer ses propositions, attendait que «l'animo » des conseillers fût «assettato », c'est-à-dire non troublé.

Le prince voulut que les membres des conseils ne fussent pas «appassionati », ce qui revient à dire qu'ils ne fussent pas entraînés outre mesure par l'adhésion, autorisée en soi, à une coalition ou à une autre ${ }^{25}$. Ludovico Sforza, un jour où il rejeta la manifestation de la volonté communautaire codifiée par la coutume - le sindacato, c'est-à-dire le document notarié qui verbalisait les travaux de l'assemblée - en faveur du rassemblement des opinions individuelles par un officier d'État, la délégitima en recourant directement à cette tradition. Il manifesta la conviction selon laquelle dans ces formes-là, il était impossible d'exprimer «en liberté » l'opinion et la volonté personnelles, écrasées par le «timore » et «l'amore», des sentiments opposés mais tout aussi négatifs l'un que l'autre, qui guidaient les comportements des individus dans les réunions plénières. À une autre occasion, il commanda le recours à une procédure de consultation analogue, vue encore comme la seule garantie possible pour que la volonté des hommes soit libre, «la voluntà di li homini sii libera », et non troublée par une sorte de régression au niveau de l'instinct.

22. E. RufFINI, La ragione dei più. Ricerche sulla storia del pensiero maggioritario, Bologne, 1977, p. $248 s q$.

23. L. ARCANGELI, «Igne et ferro. Sulle dedizioni di Reggio alla Chiesa e agli Estensi (15121523). Note a margine di alcuni studi di Odoardo Rombaldi », dans G. BADINI, A. GAMBERINI dir., Medioevo reggiano. Studi in ricordo di Odoardo Rombaldi, Milan, 2007, p. 402.

24. ASMi, CS, $1157,1497.04 .05$ et 05.21 .

25. R. Musso, «Viva el duca et lo sancto padre. Savona al tempo degli Sforza e di Sisto V (1464-1478) », Atti e memorie della Società savonese di storia patria, n.s., XXXVII, 2001, p. 59-153, p. 106. Cf. E. RovedA, Le istituzioni e la società in età visconteo-sforzesca, dans Storia di Pavia, III/1, Pavie, 1992, p. 86. 


\section{Mauvaises manières}

Comme d'autres régimes, précédents ou ultérieurs, le régime princier élabora non seulement un programme de gouvernement, mais aussi un idéal de bonnes manières, une "honestà » spécifique, apte à réguler la société politique. Même à l'âge auquel les institutions communales avaient pris leur origine et s'étaient consolidées, dans les statuts et dans les coutumes s'était dessinée la conduite appropriée pour l'assemblée, afin d'en conjurer la dégénérescence chaotique. Alors, toutefois, la forme convenable de comportement politique consistait dans l'expression verbale régulée et efficace, dans la dignité des postures, dans le respect des règles électorales ${ }^{26}$. Avec le principat furent introduits des éléments nouveaux et en partie opposés par rapport à cette tradition. La «voluntà » du prince devait être toujours obéie, elle n'avait plus besoin de convaincre et donc d'user des arts de la persuasion, et elle ne pouvait pas non plus être mise aux voix. Un code complexe de comportement public devenait ainsi obsolète, pour ne pas dire une entrave : les orateurs les plus éloquents parmi les politiciens locaux devinrent suspects aux officiers périphériques, la confrontation en conseil et les procédures de décision furent ravalées au rang d'atermoiement inutile ou de résistance voilée, en tant que facteurs de dilation de l'exécution des ordres qui arrivaient du centre. Comme le voulaient les traités de bonnes manières, pour les officiers, le discours public ne devait pas être trop long ou fastidieux, ni l'opposition animée outre mesure ${ }^{27}$. En somme, on fit consister le déroulement idéal de l'assemblée avant tout en une prompte docilité aux injonctions ducales et à la conduite des magistrats du prince, capables, avec «bon et pacifico modo », d'éviter «scandali », « confusione », « disordini », « errori ». Celui qui se soustrayait à ces engagements était, à nouveau comme dans la littérature et les traités contemporains, un «villano » étranger aux règles du savoir vivre politique.

La lettre d'un officier, en 1492, montre clairement l'usage de cet ample répertoire, moraliste et pédagogique, avec ses sources antiques et modernes, dans la polémique anti-républicaine. En écrivant au prince, le podestat de Tirano, Francesco Pasquali, fit un rapport sur l'opposition rencontrée par le projet, promu par Milan, de fortifier cette terre de confins. Un nombre restreint de «zentilhomini », choisis par le pouvoir central, avait obéi en désignant un procureur avec «pleno mandato » pour s'engager à la satisfaction de ce qui était demandé. Les "persone vulgare», au contraire, manipulées par un petit nombre d'intrigants («certi malvasi et alieni del ben vivere») résistaient et avaient décidé d'envoyer à Milan un procureur différent, «senza mandato », c'est-à-dire sans faculté de contracter de réels engagements. Pour

26. $C f$. P. Cammarosano, "L'éloquence laïque dans l'Italie communale (fin du XII ${ }^{\mathrm{e}}$ $\left.\mathrm{XIV}^{\mathrm{e}} \mathrm{s}.\right)$ », Bibliothèque de l'école des chartes, 158, 2000, p. 431-442.

27. Qu'il suffise de rappeler ici G. Della CASA, Galateo, S. Prandi éd., Turin, 1994. 
la compréhension et la description des comportements politiques des popolari, indociles et bons à rien, un passage de l'Énéide, dans son ensemble même si la citation explicite ne concernait que quelques mots, était crucial : " po acadere lo dicto de Virgilio : "saevit animis ignobile vulgus iamque faces et saxa volant" ${ }^{28}$. Les vers étaient extrapolés par une longue comparaison : « Saeditio saevitque animis ignobile vulgus, / Iamque faces et saxa volant, furor arma ministrat : / Tum pietate gravem ac meritis si forte virum quem / Conspexere, silent arrectisque auribus adstant;/ Ille regit dictis animos et pectora mulcet : / Sic cunctus pelagi cecidit fragor $»{ }^{29}$. Fort du souvenir de cet extrait, Pasquali ravalait en substance la manifestation de la volonté politique de la communauté au rang de déchaînement de la nature (la tempête marine), en contradiction avec tout idéal de dignité et de décence. Selon un topos récurrent dans les documents d'archives sforzesques, seul un homme doué de la qualité de mesure qui manquait au peuple (qui, lui, était gravis) en profitant de l'inconstance et de la malléabilité de ce dernier, pouvait le réduire au silence en en prenant la direction.

\section{La transparence et l'obstacle : la communauté devant le prince}

La tradition communale alimentait un idéal que l'on pourrait qualifier de «transparence » pour parler comme Jean Starobinski ${ }^{30}$. Les communautés revendiquaient leur droit de manifester collectivement et directement leur propre volonté, d'accéder à la présence des officiers des Sforza et du prince lui-même, éventuellement avec tous leurs membres (sans le filtre de l'écriture et sans médiations bureaucratiques). Les ambassadeurs ou les magistrats locaux à qui, normalement, étaient conférés les devoirs d'intermédiation, ne devaient pas constituer un «obstacle»; la limitation des pouvoirs qui leur étaient attribués, leur renouvellement rapide, la possibilité reconnue en théorie à chaque membre de la communauté d'assumer ces responsabilités servaient à amincir le diaphragme qu'ils constituaient entre le sujet et le sommet du pouvoir. Ainsi les hommes, grâce à leurs délégués à la cour, auraient dû se sentir idéalement "présents », « en personne », devant le duc, et admis à parler « viva voce»; la «mente », l'esprit des sujets, en somme, communiquée aux ambassadeurs, pouvait par leur intermédiaire percevoir la «mente » du prince, « cognoscere » son « intentio », dans une chaîne de communication entièrement transparente, un mouvement réciproque d'entente, «intendere l'animo».

28. ASMi, CS, 1153, 1492.02.05. ViRgile, Eneide, livres I-VI, éd. H. Goelzer, trad. A. Bellessort, Paris, 1952, L. I, v. 149-150, p. 10 : «il arrive souvent dans un grand peuple qu'une sédition éclate et que l'ignoble plèbe entre en fureur ».

29. VIRGiLE, Eneide, livres I-VI, ibid., v. 150 sq., p. 10-12: «Déjà les torches volent et les pierres ; la folie fait arme de tout. Mais alors, si un homme paraît que ses services et sa piété rendent vénérable, les furieux s'arrêtent, se taisent, dressent l'oreille: sa parole maîtrise les esprits et adoucit les cœurs. Ainsi tout le fracas de la mer est tombé. »

30. J. Starobinski, J.-J. Rousseau. La transparence et l'obstacle, Paris, 1971. 
On sait toutefois que le prince, à la Renaissance, a cultivé aussi un mythe d'inaccessibilité et s'est nimbé d'une aura d'opacité. Ce dessein est révélé pleinement par ses résidences, toujours plus éloignées dans le tissu urbain et moins faciles à pénétrer, ou les nouveaux cérémoniaux qui parfois accentuèrent le détachement par rapport aux sujets ${ }^{31}$. Sur le plan des pratiques politiques, l'organe le plus puissant du gouvernement des Sforza était le « conseil secret »; à la chancellerie, elle aussi appelée « secrète », on traitait les « archana » du duc, dont les archives se fermèrent à la libre consultation, autorisée en revanche par les communes urbaines ${ }^{32}$. Le seigneur se retira aussi face au désir de contact répandu parmi les sujets, en repoussant les ambassades trop fréquentes ou trop nombreuses des communautés à Milan, ou en les redirigeant vers son premier secrétaire.

Dans la construction d'une barrière supplémentaire, intervinrent les critères de discrimination sociale et les pratiques informelles de gouvernement dont j'ai parlé. À la cour et dans les organes suprêmes de gouvernement on ne voulait pas recevoir de délégations constituées «populariter», par des hommes humbles d'aspect («poveri di pariscentia ») et dénués de la tenue adéquate (qui ne savaient pas se comporter « reverentemente et discretamente »). On exigeait, en outre, que les corps élisent des syndics doués de l'«arbitrio » et de la faculté d'obéir « liberamente », c'est-à-dire d'obtempérer promptement aux ordres reçus sans autres consultations avec leurs mandants et donc sans la confirmation définitive de la population qu'ils représentaient ${ }^{33}$. Or la figure du médiateur d'extraction élue, choisi parfois directement d'en haut et non plus expression des assemblées de la communauté, ou encore désigné par ces organismes mais revêtu d'une autorité dégagée du mandat qu'il avait reçu, renforçait une conception plus personnelle et extra-institutionnelle du pouvoir local et de la communication entre centre et périphérie. Ainsi, à nouveau, le fait de passer par dessus la légalité communale concourait à réduire la participation des sujets à la sphère politique et aux décisions qui les concernaient.

31. P. Boucheron, «Non domus ista sed urbs: palais princiers et environnement urbain au Quattrocento (Milan, Mantoue, Urbino) », dans P. BOUCHERON, J. CHIFFOLEAU dir., Les palais dans la ville. Espaces urbains et lieux de la puissance publique dans la Méditerranée médiévale, Lyon, 2004, p. $266 s q$.

32. F. M. Vaglienti, «Fidelissimi Servitori de Consilio suo Secreto. Struttura e organizzazione del Consiglio Segreto nei primi anni del ducato di Galeazzo Maria Sforza (1466-1469) », Nuova Rivista Storica, LXXVI, 1992, p. 649 ; F. LeVERotTI, Diplomazia e governo dello stato. I «famigli cavalcanti » di Francesco Sforza (1450-1466), Pise, 1992, p. 11-13 ; EAD., "L'archivio dei Visconti signori di Milano », Reti medievali - rivista, IX, 2008, p. 12. Cf. I. LAZZARINI, «Cives vel subditi : modelli principeschi e linguaggio dei sudditi nei carteggi interni (Mantova, XV s.) », dans A. GAMBerinI, G. Petralia dir., Linguaggi politici, p. 89-112.

33. G. SCARAmELlini, Le fortificazioni sforzesche in Valtellina e Valchiavenna, Chiavenna, 2000, p. 396, doc. 370 . 


\section{La défense d'une possibilité anthropologique}

Le concours de l'État dans la promotion de l'autonomie de l'individu par rapport à la communauté et au corps social dans lequel il était inséré est un élément des lectures sociologiques et historiques les plus classiques de la mutation moderne ${ }^{34}$. Néanmoins, des perspectives renouvelées et de nouvelles techniques d'analyse peuvent donner une seconde vie à ces modèles. L'action des pouvoirs centraux dont on a ici tracé les contours eut effectivement une immense portée, qui ne peut être limitée ni à la légitimation des processus d'aristocratisation (qui, dans l'Italie de la fin du Moyen Âge, favorisèrent des élites de gouvernement plus restreintes), ni à la réorganisation de la communauté ou d'autres institutions et de leurs procédures en faveur de pratiques moins formalisées d'exercice de l'autorité. Il s'agissait aussi de produire une nouvelle anthropologie qui, avec la désinstitutionalisation partielle du politique, redéfinissait les relations interpersonnelles et le rapport entre collectivité et individu, au rôle et à la volonté duquel était conférée une plus grande autonomie.

Même si les autorités d'État n'ignoraient pas la valeur de la cohésion collective, leurs récits de la vie politique locale impliquaient de facto une déconstruction culturelle de la communauté, dont les initiatives étaient réduites à l'instigation d'un petit nombre de puissants ou de criminels ou à des manifestations atypiques, le plus souvent d'hommes de vile condition. Le choix, comme interlocuteurs, de principali forts de leur ascendance personnelle plus que de mandats juridiquement constitués, et la consultation directe des avis des «particuliers » mettaient à mal les traditions institutionnelles des corps. Mais les sujets répondirent à cette tentative de dissolution politique de la collectivité en défendant les principes de représentation et d'assemblée menacés par le discrédit des gouvernants, en soulignant de cette façon l'identité de la communauté, et la force agrégative de l'institution, capable d'induire une fusion symbolique entre ses membres.

On sait bien comment, au Moyen Âge, le savoir juridique élabora la notion de personne ficta et de corps, donnant unité et substance aux institutions associatives ${ }^{35}$. Dans ces pages, toutefois, je poursuivrai l'analyse d'un langage de la pratique politique qui connaît le droit, mais qui ne tend pas à l'élaboration doctrinale. Cette analyse implique de chercher dans les plis des écritures des corps constitués les modalités de subjectivation de la communauté et de relation entre individus qui ne sont pas assimilables à celles qui étaient proposées par les pouvoirs centraux, et pas non plus immédiatement convergentes avec les catégories analytiques de la modernité occidentale.

34. Il suffit de rappeler J. BuRCKHARDT, Die Kultur der Renaissance in Italien, Bâle, 1860, partie II.

35. P. Michaud-Quantin, Universitas. Expressions du mouvement communautaire dans le Moyen Âge latin, Paris, 1970. 
Les lettres des corps constitués affirmaient en premier lieu la supériorité du tout sur ses parties, en opposant les « tutti», et les «alcuni» ou les « certi », c'est-à-dire la commune ou l'universitas et le «particolare » ou la « singularis persona » ou les «persone private». D'autres indices font entrevoir un rapport entre individu et communauté conçu au-delà des schémas analytiques élémentaires. Dans les sources notariées et dans les archives sforzesques, les accords de nombre entre le sujet et le prédicat verbal qui devaient attribuer une action à une collectivité étaient souvent incertains ; au substantif singulier « comunità » était souvent associé un adjectif au pluriel ; en revanche, il n'était pas rare que l'individu auteur d'une lettre utilisât la première personne du pluriel, qui l'assimilait à la collectivité dont il était membre. Ainsi donc, tantôt la communauté se transformait en pluralité de ceux qui la composaient (« la comunitate [...] hanno ellezuto»), tantôt les individus se fondaient dans la communauté qui les incluait («nuy homini de questa comunitade »). En somme, l'image de la collectivité comme somme de personnes et celle de sujet unitaire se compénétraient de manière réciproque et inextricable. Un tel agrégat, pour finir, était personnifié par l'attribution d'une volonté au conseil et même à la communauté dans son ensemble (certains individus avaient ainsi agi « contra voluntà et senza consentimento de la comunità de Bormio » ${ }^{36}$ ).

Dans la Lombardie du $\mathrm{Xv}^{\mathrm{e}}$ siècle, le lieu générateur de cette volonté collective particulière était la réunion plénière des chefs de famille ou le conseil, alors que l'expression de l'opinion politique n'avait un caractère individuel qu'à l'occasion des consultations qu'on a rappelées, sollicitées directement par le prince en faveur de, ou contre, un feudataire ou un officier. Dans l'événement « appassionato » que constituait l'assemblée, les frontières entre l'opinion individuelle et la manifestation collective s'estompaient, produisant une décision institutionnelle originellement intersubjective ${ }^{37}$. En ce sens, on pourrait tenter de recomposer les différents points de vue avec lesquels sont décrites les assemblées dans les sources de l'époque, internes ou externes à la communauté. Les statuts locaux en disciplinaient le déroulement et les formules des procès verbaux ordonnaient l'enchaînement des travaux, instituant la réunion elle-même comme un moment unitaire et réglé de manifestation politique. Par contre, les lettres de quelques officiers d'État qui les présentaient comme des expériences atypiques, en faisaient des lieux de conditionnement facile des opinions d'autrui (où les voix se donnaient aussi «à l'instigation » selon Ludovic Sforza), surtout celles de personnes d'humble condition (« comuniter quilli de villa conditione se trano voluntera ad una », si l'on s'en tient à l'impression d'un religieux). Encore une fois, c'étaient les circonstances dans lesquelles, dans le cadre de l'autoreprésentation de la communauté mais aussi selon plusieurs témoignages

36. ASMi, CS, 1158, 1499.06.14.

37. Cf. L. Graham, «A Public Sphere in Amazonia ? The Depersonalized Collaborative Construction of Discourse in Xavante », American Ethnologist, 20, 1993, p. 717-741. 
externes, les hommes s'exprimaient, « omnes unanimes et concordes », « tutti concordanti ad idem velle», « tutti uniti». Que les termes soient apologétiques ou polémiques, il s'agit de toute façon de la description des moments lors desquels, à travers des passages assurément dramatiques, marqués par le heurt, la résonance et la contamination réciproque des propos, la perspective singulière était transcendée, les opinions «se trano ad una », produisant ensuite la « voluntà et consentimento » partagée des communautés, exprimée « una voce» par leurs membres et leurs acclamations.

Les mécanismes de l'ambassade et de la procuration étaient tout aussi importants pour la construction de la subjectivité institutionnelle de la communauté. Le principale choisi par le duc pour agir à la place de la population d'une terre, distingué pour sa qualité personnelle, et pour ainsi dire extrait de l'ensemble des voisins par un regard analytique porté de l'extérieur, qui, plutôt que fondre, séparait et mettait en valeur certains individus par rapport aux autres, restait, au fond, pour les institutions locales une "privata persona » qui agissait «particularmente » et à laquelle les hommes ne s'identifiaient pas. En revanche, dans les lettres écrites par les corps locaux pour le faire accréditer à la cour, l'ambassadeur élu par les conseils, est souvent resté anonyme ; non qualifié socialement, il est celui auquel les hommes se référaient avec des pronoms possessifs (« duy de li nostri »), doté du crédit et de la confiance que lui transmettait la communauté (on demandait au prince de lui donner «plena fede come a nuy stessi ${ }^{38}$ ). Mais dénué d'un pouvoir décisionnel autonome, il semble une figure dont l'identité n'est pas pleinement émancipée de celle de la collectivité dans son ensemble, à l'intérieur de laquelle elle est comme retenue. L'anacoluthe d'une lettre des conseillers du borgo de Bellano, qui passe de la troisième à la première personne du pluriel en renvoyant à des habitants qui, au nom de tous leurs voisins, furent obligés par un officier des Sforza à ne pas se rendre complices de contrebande («n'è facto comandamento per parte del locotenente de esso commissario che trei on quatro de nuy faciamo promissione che alcuna persona di questa terra non venda ne faza vendere biade, legumine né pane ad alcuni che sieno suspecti »), montre bien l'intention ou le réflexe d'assimiler la communauté et ceux qui agissaient pour le compte de cette dernière ${ }^{39}$.

Ce qu'on a dit à propos de l'assemblée, de la procuration ou de l'ambassade, implique dans les termes plus généraux la question de la représentation. Au Moyen Âge, selon les spécialistes de droit et de théologie, s'instaurait entre représenté et représentant un rapport d'identité tendancielle, qui voulait rendre effectivement présente la personne absente. Cette relation s'appliquait à la sphère économique, politique et religieuse ${ }^{40}$. Il peut être

38. Ticino ducale. Il carteggio e gli atti ufficiali, I/2, Francesco Sforza, L. Moroni Stampa, G. CHIESI éd., Bellinzona, 1994, p. 226-227, doc. 952.

39. ASMi, CS, 781, 1467.09.09.

40. H. Hofmann, Rappresentanza-rappresentazione. Parola e concetto dall'antichità all'Ottocento, Berlin, $2003^{4}$ (trad. it., Milan, 2007). 
utile, à cet égard, de vérifier à nouveau comment se positionnaient les langages de la pratique politique, dans des contextes différents de ceux de la réflexion académique. L'institution communautaire n'aurait pas pu, sans représentants, exprimer une volonté ni, plus généralement, s'affirmer comme acteur politique. Par conséquent, défendre cette possibilité contre la promotion de l'individu, classé selon sa propre qualité et agi par sa propre volonté, comme sujet agent à l'intérieur et à l'extérieur de la collectivité, était vital pour les corps locaux. Dans les écrits qui en émanent, on insistait alors sur le fait que les interventions dans les assemblées rendaient présents les absents, en conférant de l'actualité à l'universitas : trois cents «homini de la plebe de Travaglia » agissaient « representando loro la persona de tuti gli altri che li manchavano ». On voulait que les agents opérant auprès des Sforza et de leurs officiers, la rendent présente dans son unité et dans sa totalité, en modelant le rapport sur la correspondance organique entre la partie et le tout, et en rétablissant, au moins de manière rhétorique, la possibilité de cette « transparence » entre ses membres et entre ces derniers et le sommet politique dont on a parlé. Ainsi, de fait, il devenait possible que «tuta » une « universitade » parle par leur intermédiaire, et que, même lointains, les hommes dans leur ensemble se sentent face au prince («come se li fusemo presenti tuti noy »).

C'est dans ce cadre que se situe aussi la concurrence pour l'appropriation légitime du nom de la communauté. Dans le milieu et l'époque auxquels se réfèrent nos sources, le nom - signe coagulant et hypostase de l'identité individuelle ou collective - avait un rôle fondamental dans les processus de production du sujet même qui le portait. Les personnes respectables étaient celles qui «porteno... [...] bono nome ». Même le rôle était un nom, si l'on dénonçait les malversations dont se rendaient coupables les « officiali sotto il nome e scudo de comissarii » (c'est-à-dire à qui était conférée cette dignité spéciale) ${ }^{41}$. Quand les foules s'exprimaient pour ou contre le régime ou proclamaient leur adhésion à une coalition, invoquer le nom du prince ou du chef de parti signifiait exhiber le signe distinctif du groupe. Ce n'est pas un hasard si les pouvoirs centraux, pour désagréger la loyauté de faction, interdirent entre autre de prononcer les noms mêmes de guelfe, gibelin, ou de tout autre parti local, comme si taire le nom condamnait le parti à l'inexistence $^{42}$. La communauté aussi recevait une sorte de baptême : c'est-à-dire qu'elle avait un nom dont dépendait son engagement dans un rôle politique en tant que tel. L'identification avec la collectivité, en effet, pouvait être revendiquée ou contestée par des groupes locaux en compétition, par exemple

41. M. Della Misericordia, «Per non privarci de nostre raxone »..., p. 209.

42. R. M. Dessì, «I nomi dei guelfi e ghibellini da Carlo d'Angiò a Petrarca », et M. GENTILE, «Postquam malignitates temporum hec nobis dedere nomina ... Fazioni, idiomi politici e pratiche di governo nella tarda età viscontea», dans M. GENTILE dir., Guelfi e ghibellini nell'Italia del Rinascimento, Rome, 2005, p. 3-78, 249-274. 
lorsqu'on accusait le représentant d'avoir agi non pas «nomine communis, sed quorundam particularium personarum ». Elle pouvait surtout se voir opposée à la perspective réductionniste des officiers d'État: un commissaire révéla par exemple les responsabilités personnelles de quelques nobles, désignés par leur nom, prénom, titre honorifique et degré de parenté, qui au contraire faisait retomber les fautes sur la communauté. Dans ses mots, l'institution restait uniquement le nom derrière lequel se cachaient les figures des principali ( licet se batizano la comunità, tamen se resolveno \& concludeno in alchuni »). Pour ces raisons, l'usage légitime du nom de la collectivité était exhibé dans les écritures et en même temps surveillé au moyen de procédures institutionnelles transmises par la coutume : dans l'assemblée des chefs de famille et des conseils, on décidait «nomine et vice ipsius communis »; les officiers locaux opéraient «nomine communis »; et l'on écrivait au prince «in nome de tuta la comunità ». Les ambassadeurs, pour finir, dans les lettres de créance qu'ils présentaient au duc, n'étaient, bien souvent, désignés ni par leur nom ni par aucune indication concernant leur qualité - ce qui contredisait ouvertement l'attitude des autorités d'État -, de sorte qu'ils agissaient exclusivement «sub nomine communis».

Traduit de l'italien par Laurence MoulinIER-BROGI

Massimo Della Misericordia, Università degli Studi di Milano Bicocca, Dipartimento di scienze umane per la formazione « Riccardo Massa », Piazza dell'Ateneo nuovo 1 (Edificio U6, IV piano), I-20126 Milano

\section{Principat, communauté et individu au bas Moyen Âge. Cultures politiques dans l'État de Milan}

Dans la Lombardie de la fin du Moyen Âge, un débat politique animé concernait la légitimité des corps territoriaux. La rhétorique de la principauté attaqua les valeurs de la tradition communale qui s'était développée dans la cité et la campagne depuis le XIII ${ }^{\mathrm{e}}$ siècle : l'unité de la communauté, le profil institutionnel de ses structures et l'égalité idéale parmi ses membres. Les officiers ducaux critiquèrent la nature chaotique et les passions négatives de la vie publique locale. D'un autre côté, la distinction sociale et le rôle public des individus (particulièrement des leaders locaux) furent promus. À l'opposé, les corps territoriaux défendirent avec succès les pratiques et le modèle de la cohésion de la communauté, dans la décision politique et la représentation, et insistèrent sur l'identité collective de la population de la ville ou du village.

État de la Renaissance - communauté - identité politique collective - identité individuelle - distinction sociale - représentation politique 
Princedom, Community and the Individual in the Late Middle Ages. Political Cultures in the State of Milan

In the Lombardy of the late middle Ages an animate political debate concerned the legitimacy of territorial bodies. The rhetoric of principality attacked the values of the communal tradition that since the 13th century developed in the city and in the country : the unity of community, the institutional profile of its structures and the ideal equality among its members. Ducal officers criticized the chaotic nature and the negative passions of local public life. On the other hand, the social distinction and the public role of the individuals (particularly of local leaders) were promoted. On the contrary, territorial bodies defended with success the practices and the model of community cohesion, in political decision and representation, and always stressed the collective identity of village or town population.

Renaissance state - community - collective political identity - individual identity - social distinction - political representation 
